\section{TRYPANOSOMIASIS IN A TIGER (PANTHERA TIGRIS)}

\author{
S.V. Upadhye ${ }^{1}$ and V.M. Dhoot ${ }^{2}$ \\ ${ }^{1}$ Assistant Professor (Veterinary Science), College of Agriculture, \\ Nagpur, Maharashtra, India \\ ${ }^{2}$ Officer-in-charge, Maharajbag Zoo, College of Agriculture, \\ Nagpur, Maharashtra, India.
}

Surra caused by Trypanosoma evansi is a common disease widely reported in domestic animals. However, because of its acute and fatal nature, the reports on Trypanosomiasis in wild animals are very few (Sinha et al., 1971; Choudary et al., 1986). The present communication reports a case of Trypanosomiasis in a Tiger and its successful treatment.

A male adult tiger Chandu aged 9 years at Maharajbag Zoo, Nagpur was observed ill for two days with symptoms of anorexia, constipation and lethargy. There was rapid respiration, panting and with the inclination to press the head on the bars of the cage. The Tiger had convulsions once in the early morning. The efforts to give antipyretic/analgesic drugs orally with food and water failed as the tiger did not show interest in the food. Then the Tiger was shifted into a squeeze cage for closer clinical examination. Pulse was rapid (80/min) and body temperature was $\left(106.8^{0} \mathrm{~F}\right)$. The blood smears were sent to a laboratory for examination. Meanwhile, injections of Diclofenac sodium (10 ml.) and B-complex $(8 \mathrm{ml}$.) were administered to the Tiger intramuscularly. The blood smear was found positive for Trypanosoma evansi infection. Berenil (total dose of 3 g.) was immediately given intramuscularly on the same day. Since the Tiger was suspected to be hypoglycemic, an injection of Dextrose $10 \% 500 \mathrm{ml}$. was given intravenously. The injections of Diclofenac sodium and B-complex were repeated for one day and three days respectively. The tiger was kept under close observations for about a week.

The temperature recorded eight hours after treatment showed decline $\left(103.8^{\circ} \mathrm{F}\right)$ and the animal gradually returned to normal on the second evening. The Tiger accepted some water on the same evening but did not show any interest in solid food. His appetite was fully restored on the fifth day after treatment with evidences of normal respiration and cessation of nasal discharge.

The head pressing or convulsions were not noticed after the beginning of the treatment. The Tiger showed complete recovery and was released in to the enclosure on the sixth day.

Sporadic cases as well as outbreaks of trypanosomiasis have been reported in tigers. Sinha et al. (1971) reported on outbreak of surra in four Tigers, two Jaguars and one Leopard. He reported sudden deaths in four animals without any signs of illness whereas three animals showed respiratory distress, running nose in one and convulsive fits in one animal. Reddy (1975) reported the successful treatment of an outbreak of surra in circus tigers. The major sign of the disease was pyrexia $\left(106^{0} \mathrm{~F}-108^{\mathrm{O}} \mathrm{F}\right)$. No other signs were noticed. Choudhary et al. (1986) reported a sporadic case of surra in a tiger which died suddenly with fever. He reported trypanosomes in the heart blood smear. In the present case, the Tiger suffered from the signs such as anorexia, pyrexia $\left(106.8^{\mathrm{O}} \mathrm{F}\right)$, nasal discharge with respiratory distress and head pressing. The authors also observed convulsions only once but not after the administration of Berenil.

Prompt treatment with Berenil and B-complex (Livobex) was undertaken as suggested by Reddy (1975). Similarly, considering anorexia and possible hypoglycemia in surra, $10 \%$ Dextrose was also given to combat hypoglycemia. Laha et al. (1991) also reported hypoglycaemia in trypanosomiasis in buffalo calves. In his opinion hypoglycemia could be due to consumption of glucose by haemoprotozoa or disturbance in carbohydrate metabolism of the host. Only single injection of Berenil gave excellent result and the blood smear taken on $6^{\text {th }}$ post treatment day did not reveal the pathogens and hence no further treatment was thought necessary. The Tiger showed complete and uneventful recovery.

\section{References}

Choudary, C., B. Narasimhaswamy, J. Hararamdas, P.B. Rao and M.R.K. Rao (1986). A case of sudden death in a male tiger cub (Panthera tigris) with Trypanosomiasis - a note. Indian Vet. J. 63(6): 506-507.

Reddy, R.G., D.R. Sharma and Ch. Choudary (1975). An outbreak of surra in circus tigers. Indian Vet. J. 52: 406.

Laha, R., K.D. Prasad and B.N. Sahai (1991). Status of blood glucose during $T$. evensi infection and quinapyramine prosalt treatment in baffalo calves. Indian Vet. J. 68: 311-313.

Sinha, P.K., G.S. Mukherjee, M.S. Das and R.K. Lahiri (1971). Outbreak of Trypanosomiasis evansi amongst tigers and jaguars in the Zoological Garden, Calcutta. Indian Vet. J. 48: 306 